\title{
ACUMULACIONES FLUVIALES EN LA DEPRESIÓN DEL EBRO: VALORACIÓN DE LA VALIDEZ DE UNA SECUENCIA GENERAL
}

\author{
Asunción JULIÁN ANDRÉS y Javier CHUECA CÍA \\ Departamento de Geografía y Ordenación del Territorio \\ Universidad de Zaragoza
}

\begin{abstract}
Resumen: En este trabajo se relacionan las secuencias de terrazas fluviales identificadas para los principales cursos de la Depresión del Ebro así como los datos de cronología absoluta y relativa disponibles hasta el momento. Se analizan algunos de los factores fundamentales en la configuración y conservación de estas morfologías y se cuestiona la validez de una correlación general de acumulaciones fluviales para toda la cuenca a partir de la información actual disponible.
\end{abstract}

Palabras clave: Terrazas fluviales, secuencias evolutivas, correlación general, Depresión del Ebro.

\begin{abstract}
In this work the sequence of fluvial terraces identified in the main courses of the Ebro Basin are exposed as well as the absolute and relative chronology data available at the present moment. Some fundamental factors in the conformation and conservation of these morphologies are analyzed, and the validity of a global correlation of fluvial accumulations for the basin from current available information is discussed.
\end{abstract}

Key words: Fluvial terraces, evolutive sequences, global correlation, Ebro Basin.

\section{INTRODUCCIÓN}

Más de una tercera parte de la Depresión terciaria del Ebro se halla recubierta por acumulaciones cuaternarias, siendo las terrazas fluviales las morfologías que mayor interés han acaparado entre los estudiosos del relieve (Fig. 1). Sin embargo, son muy escasos los trabajos que ofrecen una visión de conjunto de un amplio sector de la cuenca, de manera que la mayor parte de ellos constituyen investigaciones puntuales, o en todo caso referidas a sectores más o menos extensos, pero sin una intención clara de correlacionar sus resultados.

Dentro de los análisis que han contribuido a la cartografía e identificación de los distintos sistemas de terrazas en la Depresión del Ebro, cabe resaltar por el carácter 
general que revisten los de BOMER (1978), relativo a la geomofología de toda la Depresión del Ebro y JULIÁN (1997), centrado en las acumulaciones cuaternarias de este mismo ámbito. Algo más concretos son los de MENSUA e IBÁÑEZ (1977) y ALBERTO et al., (1984), referidos al Cuaternario de la porción aragonesa de la Depresión. Igualmente destacable es todo el conjunto de Memorias de Licenciatura y Tesis Doctorales realizadas por geógrafos y geólogos de la Universidad de Zaragoza donde se estudia el relieve de buena parte de la Depresión del Ebro: IBÁÑEZ (1976), sobre el Piedemonte Ibérico Bajoaragonés; ZUIDAM (1976), relativa a los alrededores de Zaragoza; YETANO (1978), sobre el Valle del Huerva; GONZALO (1981), referida a La Rioja; PEÑA (1983), que estudia el sector comprendido entre los ríos Segre y Noguera Ribagorzana; ECHEVERRÍA (1984), relativa al valle del río Aguasvivas; PELLICER (1984), sobre la geomorfología de las Cadenas Tbéricas entre el Jalón y el Moncayo; IBARRA (1985), sobre el río Arba de Biel; GRACIA (1985), relativa a las Bardenas Orientales; RODRÍGUEZ (1986), centrada en el Somontano pirenaico oscense; SORIANO (1986), sobre la región centromeridional de la Depresión del Ebro entre los ríos Jalón y Ginel; SANCHO (1988), sobre la cuenca baja del río Cinca; BENITO (1989) referida a la cuenca baja del río Gállego; o LERÁNOZ (1993), a propósito de La Ribera de Navarra.

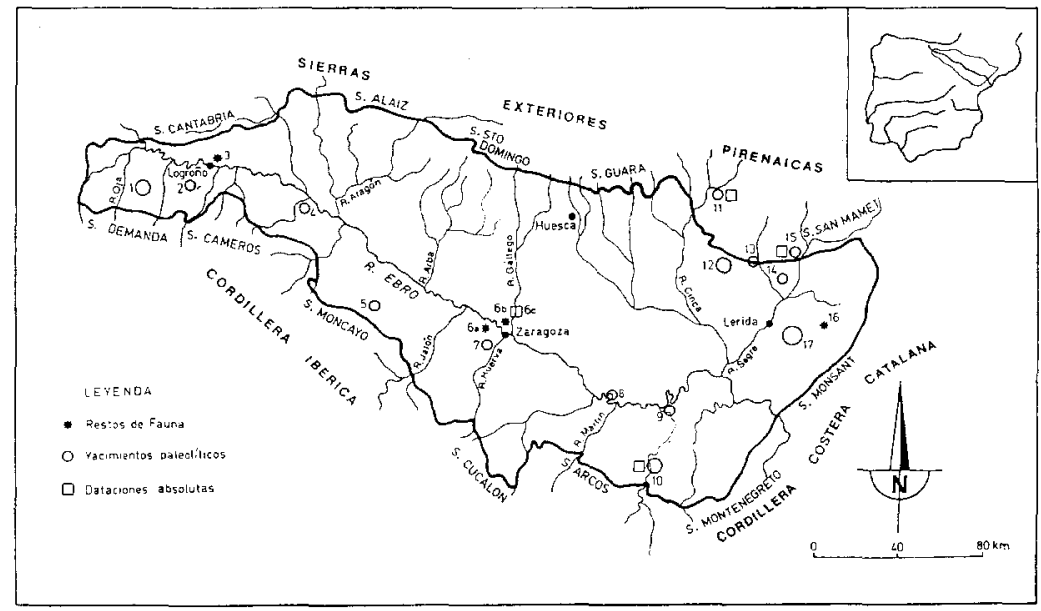

Figura 1.- Localización en el ámbito de la Depresión del Ebro de restos de fauna, yacimientos paleolíticos y depósitos pleistocenos con dataciones absolutas.

1) Conjunto del Najerilla; 2) Navarrete; 3) Cerro de Cantabria; 4) Conjunto de Calahorra;

5) La Bardalera; 6 a-b) Conjunto Ebro-Gállego; 6c) Dataciones paleomagnéticas en sector Gurrea-Zaragoza; 7) Cadrete; 8) Sástago; 9) Caspe; 10) Castelserás; 11) Las Forcas; 12)

Conjunto de La Litera; 13) Cueva de l'Estret de Tragó; 14) Conjunto del Farfanya; 15) La Roca dels Bous; 16) Miralcamp; 17) Conjunto del Set y Femosa. 
En este artículo, en definitiva, intentaremos valorar las posibilidades de establecer una secuencia general de las acumulaciones cuaternarias, válida para toda la Depresión del Ebro, a partir de la información disponible en el momento actual, información que engloba los datos citados en las monografías anteriores y los aportados por otras de carácter más temático o puntual.

\section{DISTRIBUCIÓN ESPACIAL Y MORFOLOGÍA DE LAS ACUMULACIONES FLUVIALES EN LA DEPRESIÓN DEL EBRO}

La mayor o menor extensión que presentan las acumulaciones fluviales de la Depresión del Ebro se halla condicionada por la dinámica de cada curso que, a su vez, depende estrechamente de factores morfoestructurales; esto determina que su distribución espacial manifieste notables irregularidades entre los distintos sectores de la zona de estudio (JULIÁN, 1997). Así, en el área occidental riojana los mantos aluviales de mayor extensión pertenecen a los sistemas fluviales de procedencia ibérica -ríos Oja, Najerilla, Iregua y Leza-, y las terrazas vinculadas al río Ebro tan sólo adquieren una importancia significativa aguas abajo de Logroño. Por el contrario, en el sector central las mayores superficies acumulativas pertenecen al río Ebro y a los cursos pirenaicos (Arbas en Las Cinco Villas, Flumen-Alcanadre en la Depresión de Sariñena, Gállego y Cinca-Segre), con una capacidad de descarga muy superior a los de origen ibérico. Ya en el sector oriental, los sistemas fluviales que han generado mantos aluviales de cierta importacia tienen sus áreas de cabecera en las sierras pirenaicas (ríos Cinca, Segre y Noguera Ribagorzana) o en los relieves monoclinales de La Segarra y Les Garrigues (Sió, Ondara, Corb, Femosa y Set).

Respecto a la morfología adoptada por las terrazas, en función de la adaptación de los distintos cauces al relieve diferencial heredado, pueden establecerse tres modelos básicos (JULIÁN, 1997): 1) acumulaciones con morfología en abanico y amplias llanuras aluviales, correspondientes a uno o varios cursos muy divagantes, y cuyos ejemplos más significativos se encuentran en los principales ríos ibéricos riojanos (Oja y Najerilla) y en las depresiones presomontanas pirenaicas (Arbas, Flumen, Guatizalema y Alcanadre); 2) acumulaciones fluviales en banquetas adosadas a los márgenes del cauce (ríos Ebro, Jalón, Huerva, Arga, Segre, etc.), propias de cursos más confinados, aunque también puedan registrarse en ellos ciertos desplazamientos; 3) dentro de estas últimas, aunque representado un subtipo peculiar, estarian las acumulaciones características de los cauces meandriformes encajados, magníficamente representadas en los tramos occidental y oriental del río Ebro. 


\section{SECUENCIA DE LAS TERRAZAS FLUVIALES EN LA DEPRESIÓN DEL EBRO}

\section{Niveles acumulativos}

El número de niveles de acumulación diferenciables en los distintos sistemas aluviales de la Depresión del Ebro oscila entre tres y doce (incluyendo el nivel holoceno), localizándose a unas alturas relativas respecto al cauce actual entre los 2-3 y los 245 m (JULIÁN, 1997). Sin embargo, los niveles superiores tan sólo aparecen representados puntualmente, siendo las secuencias más habituales dentro de los principales sistemas las de ocho o nueve episodios. Por encima de estos niveles se dispone una serie de depósitos a unas alturas relativas que suelen rebasar los $250 \mathrm{~m}$ tradicionalmente considerados como pliocuaternarios, aunque no haya una base cronológica firme para afirmarlo-, de aspecto residual y alejados espacialmente respecto a los cauces actualmente funcionales, características que dificultan su inclusión dentro de una secuencia general.

Los datos utilizados en este trabajo en relación al número de niveles acumulativos y a las alturas a las que se hallan los mismos corresponden: al río Ebro, en el que diferenciamos varios tramos, a los ríos de procedencia ibérica más significativos (Tirón, Oja, Najerilla, Iregua, Leza, Cidacos, Alhama, Queiles, Huecha, Jalón, Huerva, Aguasvivas, Martín y Guadalope), y a los principales cursos pirenaicos (Ega, Arga, Aragón, Zidacos, Arba, Gállego, Sotón, Isuela, Flumen, Guatizalema, Alcanadre, Vero, Cinca, Noguera Ribagorzana y Segre).

1) El Ebro presenta un sistema acumulativo de nueve niveles en el sector riojano (JULIÁN, 1997), once en el sector navarro (LERÁNOZ, 1993), ocho en el sector central zaragozano (SORIANO, 1990), y nueve en el bajoaragonés (JULIÁN 1996), oscilando sus alturas relativas entre 1 y $220 \mathrm{~m}$ (Fig. 2). Este sistema, a pesar de constituir el nivel de base principal de la red de drenaje de la Depresión, no registra por tanto, ni la secuencia más completa, ni los niveles de terraza con cotas más elevadas.

2) En el sector del piedemonte ibérico la serie más amplia de niveles acumulativos corresponde a los ríos Oja y Najerilla $\left(T_{1}-T_{10}\right)$ y al Iregua $\left(T_{1}-T_{9}\right)$, situándose los más altos a $245 \mathrm{~m}$ en el caso del Najerilla (niveles de Valpierres y Salinillas), y a $235 \mathrm{~m}$ en el caso del Iregua (nivel del Pico del Águila) (JULIÁN, 1997) (Fig. 3). Ya en tierras aragonesas, los cursos fluviales presentan, en general, sistemas de terrazas muy limitados, tanto por la reducida extensión ocupada por las acumulaciones, como por el escaso número de niveles diferenciables. Tan sólo en el caso del Jalón y el Huerva (ambos con ocho niveles acumulativos; SORIANO, 1990), dichos sistemas han alcanzado un desarrollo considerable. 


\section{RÍO EBRO}

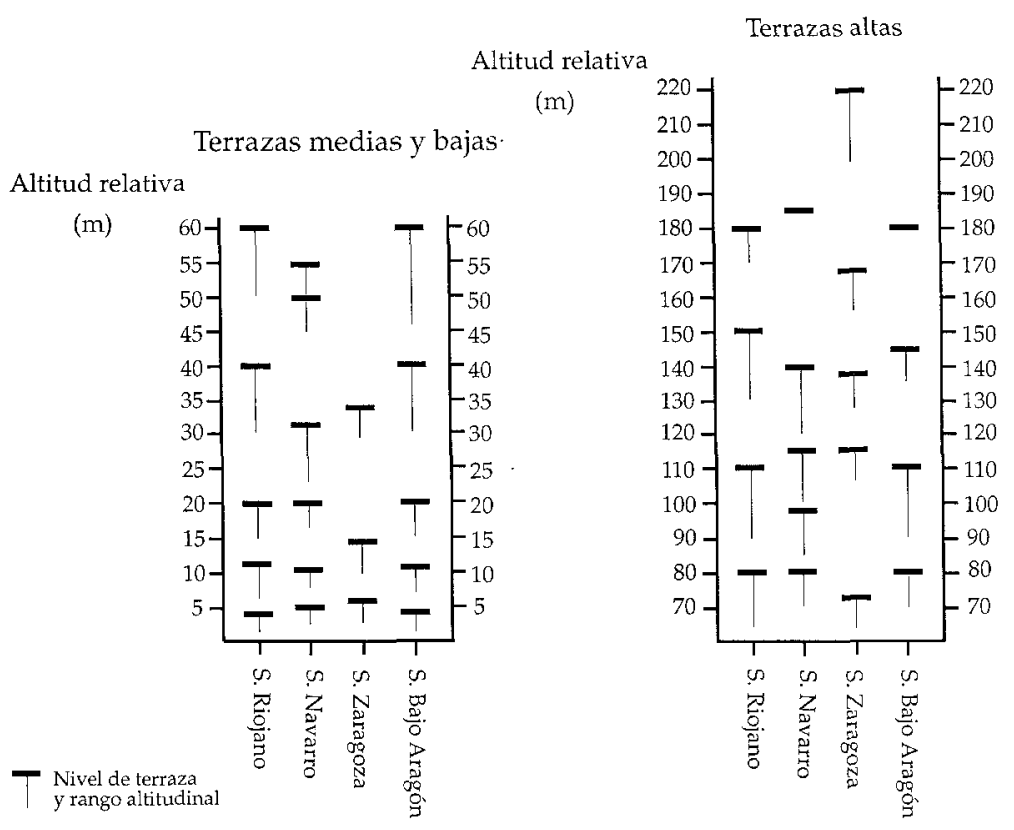

Figura 2.- Secuencias de niveles de terrazas del río Ebro en varios tramos de la Depresión.

Fuente de los datos de altitud relativa: sector Riojano JULIÁN (1997); sector navarro

LERÁNOZ (1993); sector Zaragoza SORIANO (1986); sector Bajo Aragón JULIÁN (1996).

3) En el piedemonte pirenaico occidental la secuencia más completa es la del río Aragón, con nueve niveles cuyas alturas relativas oscilan entre los 2 y los $170 \mathrm{~m}$ (LERÁNOZ, 1992) (Fig. 4). En el sector central el principal colector es el Gállego que, según las revisiones más recientes, ha desarrollado un sistema de doce niveles escalonados entre los 2 y los $175 \mathrm{~m}$ (BENITO et al., 1998). En los somontanos de Ayerbe, Huesca y Barbastro las terrazas, aprovechando un dispositivo litoestructural favorable, alcanzan un amplio desarrollo superficial; sin embargo, las secuencias más completas están compuestas por tan sólo cinco niveles (ríos Guatizalema y Alcanadre) (RODRÍGUEZ, 1986; SANCHO, 1988). Por último, en el sector oriental de la Depresión del Ebro las acumulaciones fluviales ligadas al río Cinca han sido ordenadas en la que constituye hasta el momento una de las secuencias más completa de toda la Depresión del Ebro, con once niveles dispuestos entre los 2 y los $200 \mathrm{~m}$. E1 Noguera Ribagorzana y Segre presentan sistemas algo más limitados, con siete niveles para el primero de ellos y seis para el segundo (PEÑA, 1988; PEÑA y SANCHO, 1988). 


\section{RÍOS IBÉRICOS \\ Terrazas altas}

Altitud relativa

(m)

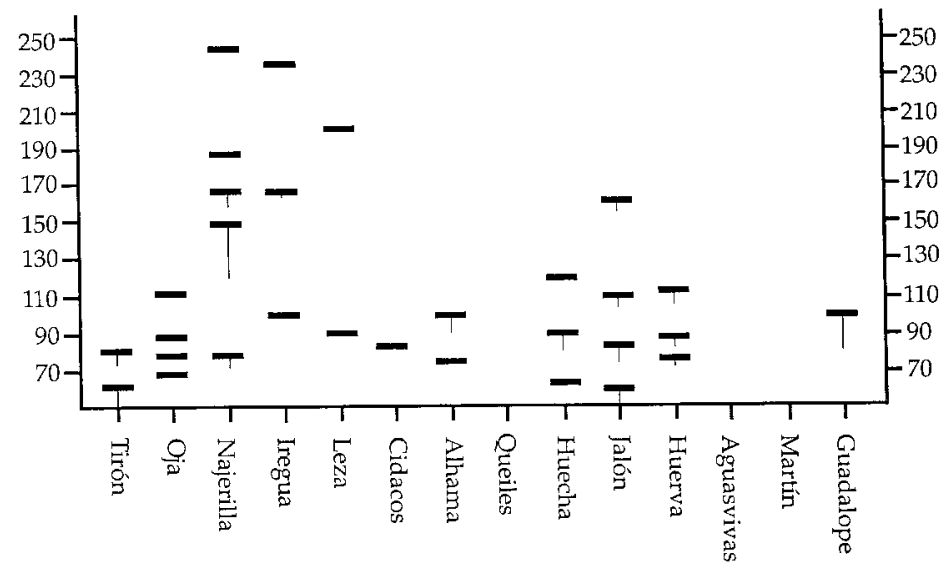

\section{RÍOS IBÉRICOS}

Terrazas bajas y medias

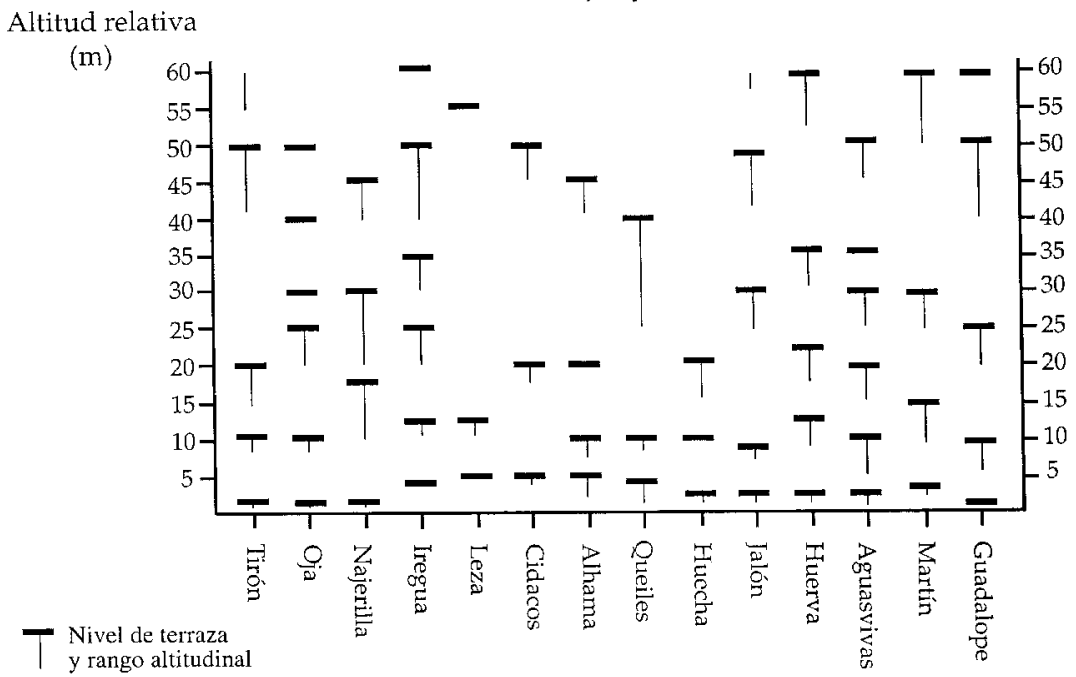

Figura 3.- Secuencias de niveles de terrazas de los principales ríos ibéricos en la Depresión del Ebro. Fuente de los datos de altitud relativa: Tirón, Oja, Najerilla e Iregua, JULIÂN (1997); Leza y Cidacos, GONZALO (1981); Alhama y Queiles, LERÁNOZ (1993); Huecha, PELLICER (1984); Jalón y Huerva, SORIANO (1986); Aguasvivas, ECHEVERRÍA . (1984); Martín y Guadalope, JULIÁN (1996). 


\section{RÍOS PIRENAICOS \\ Terrazas altas}

Altitud relativa

(m)

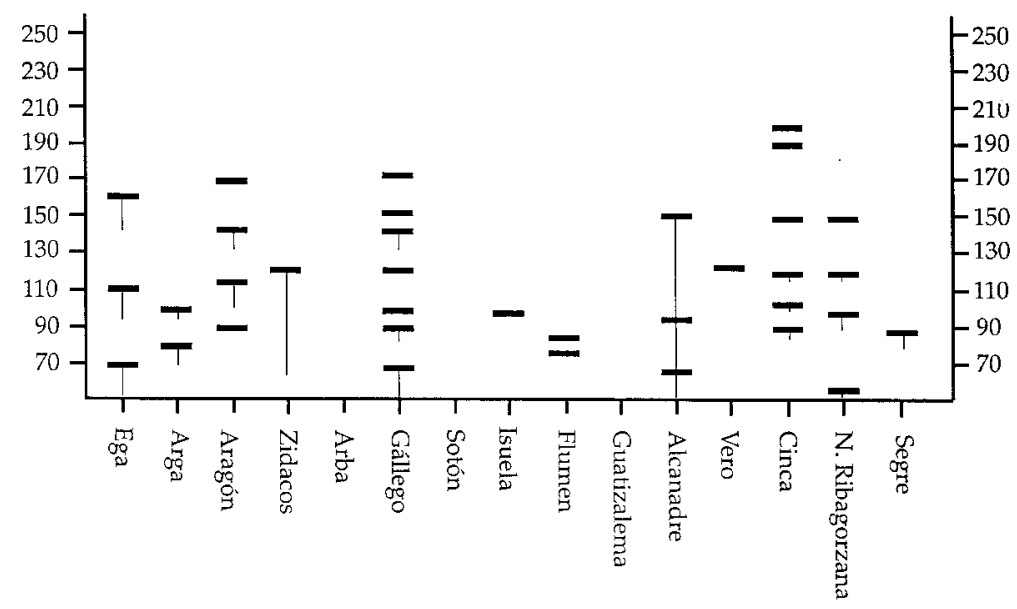

\section{RÍOS PIRENAICOS}

Altitud relativa

Terrazas bajas y medias

(m)

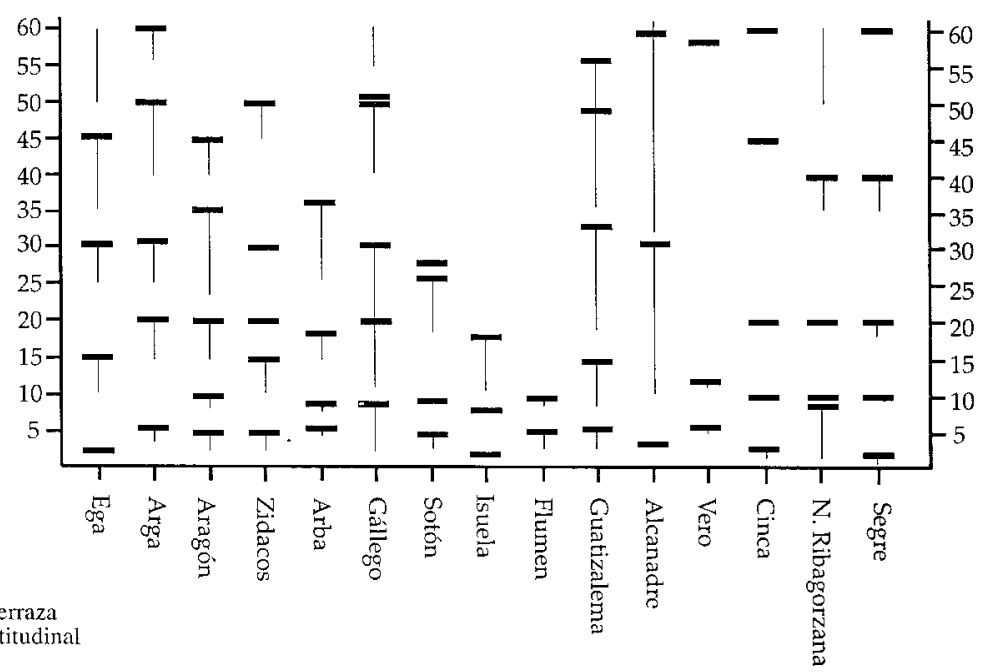

Figura 4.- Secuencias de niveles de terrazas de los principales ríos pirenaicos en la Depresión del Ebro. Fuente de los datos de altitud relativa: Ega, Arga, Aragón y Zidacos, LERÁNOZ (1993); Arba, GRACIA (1985); Gállego, BENITO, G., PÉREZ-GO̊NZÁLEZ, A., GUTIÉRREZ, F. y MACHADO, M. J. (1998); Sotón, BENITO (1989); Isuela, Flumen,

Alcanadre y Vero, RODRÍGUEZ (1986); Cinca, Noguera Ribagorzana y Segre, PEÑA y SANCHO (1988). 


\section{Datos cronológicos}

Probablemente en estos momentos, uno de los principales problemas en relación con el estudio de las acumulaciones fluviales de la Depresión del Ebro es la escasez de dataciones absolutas o relativas que permitan llevar a cabo un ordenamiento temporal preciso de las distintas secuencias deposicionales identificadas. En este apartado haremos un breve repaso a los datos cronológicos más significativos de los que se dispone en la actualidad.

Respecto a las dataciones relativas basadas en el hallazgo de restos de fauna englobados en los depósitos de las acumulaciones, las referencias de las que disponemos son sumamente escasas y no pueden ser utilizadas como un elemento de datación satisfactorio (Fig. 1). En las proximidades de Logroño (Cerro de Cantabria, correspondiente con la $T_{8}$ del río Ebro), LÓPEZ AGOS $(1948,1965)$ localizó algunos restos de elefántidos (molares y fragmentos de mandíbulas) atribuidos a Elephas antiquus falc. y Elephas meridionalis nesti, pero la escasa significación de estos fragmentos óseos (con visibles huellas de rodamiento), hizo que ya incluso en aquellos momentos se descartara su valor cronológico. También de dudosa fiabilidad son los restos de Elephas meridionalis (varios fragmentos de defensas) encontrados en la $T_{3}$ $(15 \mathrm{~m}$ ) del río Ebro en los alrededores de Zaragoza (Garrapinillos) (ZUIDAM et al., 1975), así como los de Bos primigenius y Equus caballus que aparecieron en el sector de Miralcamp, dentro de los Llanos de Urgell, en el nivel de terraza $\mathrm{T}_{6}$ (BATALLER, 1937). Por otra parte, ni los restos de Elephas antiquus localizados por OBERMAIER (1925) en una terraza del Gállego en los alrededores de Villanueva, ni los recientemente descubiertos de Elephas meridionalis en un sector muy próximo a éste, aportan una información de carácter cronológico demasiado útil, dada la dificultad de establecer la altura relativa de los depósitos en los que se engloban dentro del contexto sedimentario general.

Uno de los métodos que, en otras cuencas peninsulares, ha proporcionado una secuencia cronológica bastante completa en relación con las acumulaciones cuaternarias es el geoarqueológico, a través del hallazgo de industrias prehistóricas englobadas en los depósitos. Lamentablemente, por el momento -y a pesar de que las labores de prospección han sido ya intensas en bastantes sectores-, los yacimientos paleolíticos localizados en la Depresión del Ebro en relación con terrazas son muy escasos y además, en muchas ocasiones, los restos arqueológicos se han recogido en la superficie de las acumulaciones y no en su interior lo que, en gran medida, invalida su uso como método de datación.

Si nos limitamos a los hallazgos encontrados en el interior de los depósitos únicamente contamos con seis referencias (Fig. 1), de las cuales tres apenas son significativas: las industrias del Paleolítico Inferior y Medio que según SÁEZ (1955) 
se habrían recuperado en una de las terrazas medias del río Huerva (terraza de 30-35 $\mathrm{m})$, pero cuya existencia y ubicación no ha podido ser constatada en la actualidad; el magnífico bifaz amigdaloide de Caspe $\left(\mathrm{T}_{3}\right.$, a $\left.15-20 \mathrm{~m}\right)$, que poca información aporta a 1 tratarse de un elemento aislado sin una clara adscripción cultural (MAZO y UTRILLA, 1992); y el núcleo y las lascas encontradas en las terrazas de 19 y $12 \mathrm{~m}$ del río Guadalope (MACKLIN y PASSMORE, 1995; UTRILLA y TILO, 1995), que tampoco permiten afirmar con rotundidad en qué tipo de industria se enmarcan.

Los únicos hallazgos que reúnen suficientes requisitos para considerarse como criterio cronologico son: el de Las Forcas, yacimiento Magdaleniense relacionado con la terraza $\mathrm{T}_{2}$ (8-10 m) del río Ésera (UTRILLA y MAZO, 1991); el de la cueva de L'Estret de Tragó, industria musteriense que apareció en el interior de un depósito fluvial identificado como la terraza $\mathrm{T}_{4}(50-55 \mathrm{~m}$ ) del río Noguera Ribagorzana (MORA et al., 1992); y el de La Roca dels Bous, yacimiento atribuido también al Paleolítico Medio (Musteriense), y vinculado con la terraza $\mathrm{T}_{3}(15 \mathrm{~m})$ del río Segre (CARBONELL et al., 1987).

Respecto a las dataciones absolutas en el ámbito de la cuenca del Ebro son prácticamente inexistentes, y las únicas referencias con las que contamos corresponden a los ríos Guadalope, Ésera, Segre y Gállego (Fig. 1).

En el río Guadalope, MACKLIN y PASSMORE (1995) establecen una secuencia de once terrazas aluviales, escalonadas entre $\operatorname{los} 4$ y $\operatorname{los} 81 \mathrm{~m}$ de altitud relativa con respecto al cauce actual, y realizan en ellas varias dataciones mediante el método IRSL, obteniendo una cronología de $115.000 \pm 17.000 \mathrm{BP}$ para la $\mathrm{T}_{6}(23 \mathrm{~m})$, y de 28.000 4 4.000 BP para la $\mathrm{T}_{9}(12 \mathrm{~m})$. Para el Ésera, las dataciones proceden del ya mencionado yacimiento arqueológico del abrigo de las Forcas, en el que los niveles vinculados con la $\mathrm{T}_{2}(8-10 \mathrm{~m})$ del Ésera han sido datados mediante $\mathrm{C}_{14}$ entre $12.620 \pm$ $380 / 360$ BP y $13.010 \pm 320 / 310$ BP (UTRILLA, 1995). Y en relación con el Segre, en el yacimiento del abrigo de La Roca dels Bous, los niveles situados sobre la terraza $T_{3}(15$ $\mathrm{m}$ ) registran una cronología, también obtenida a partir de $\mathrm{C}_{14}$, de $38.800 \pm 1.200 \mathrm{BP}$ para la unidad superior, y de $>46.900$ BP para la unidad inferior (FULLOLA, 1992). Por último, recientemente se ha utilizado la magnetoestratigrafía en las terrazas del río Gállego en su sector de confluencia con el Ebro (BENITO et al., 1998) aunque, por el momento, la secuencia obtenida no permite realizar grandes precisiones, diferenciándose únicamente dos series de terrazas: las correspondientes a la época de polaridad inversa Matuyama (pre-780.000 BP, terrazas con alturas desde los $85-90 \mathrm{~m} \mathrm{y}$ superiores), y las englobadas dentro de la época de polaridad normal Brunhes (post$780.000 \mathrm{BP}$, terrazas con alturas desde los 60-70 $\mathrm{m}$ hasta el nivel holoceno). 


\section{VALORACIÓN DE LAS POSIBILIDADES DE CORRELACIÓN: HIPÓTESIS EXPLICATIVAS}

Tradicionalmente en la literatura geomorfológica se han definido tres tipos de terrazas fluviales: climáticas, tectónicas y de respuesta compleja (SCHUMM, 1981; BULL, 1990). El planteamiento de una correlación de niveles de acumulaciones fluviales, especialmente cuando se refiere a amplios sectores, implica que se asume como hipótesis genética básica la climática, es decir, la vinculación de los procesos de sedimentación e incisión con la alternancia de distintintos períodos climáticos. Sin embargo, algunos autores inciden en la importancia que pueden revestir factores de carácter local y dudan de la posibilidad de establecer una correspondencia entre los cambios climáticos globales y el desarrollo de los rellenos aluviales (PATTON y SCHUMM, 1981; PETTS y FOSTER, 1985).

De hecho, en la Península Ibérica, la complejidad a la hora de establecer un modelo evolutivo general para grandes cuencas ha sido puesta de manifiesto en el caso de la cuenca del Duero (PÉREZ-GONZÁLEZ, 1989) y especialmente en la del Tajo, donde PERÉZ-GONZÁLEZ et al., (1989) consideran que la superposición de la tectónica y la naturaleza litológica del sustrato al factor climático, ha determinado la gran variabilidad existente tanto en la morfología como en el número de terrazas identificadas en los distintos sectores. En el ámbito de la Depresión del Ebro consideramos, igualmente, que el factor climático ha jugado un papel importante en el desarrollo de etapas de acumulación-incisión, aunque debe aceptarse igualmente la intervención más o menos local -en determinadas cuencas puede ser muy llamativa- de otros controles como la tectónica, el tipo de litología, la dinámica de los cursos fluviales o, en el caso de las acumulaciones holocenas más recientes, de la propia acción antrópica.

Tradicionalmente, y ante la escasez de otro tipo de datos de valor cronológico, el criterio fundamental seguido por los investigadores que han trabajado en la Depresión del Ebro a la hora de acometer una correlación general de terrazas ha sido el establecer la altitud relativa de dichas acumulaciones en relación con la del cauce actual de cada curso. La representación en una misma gráfica de las terrazas de las ríos más significativos de la Depresión con sus correspondientes alturas (afluentes ibéricos, pirenaicos y el río Ebro en cuatro sectores), permite constatar varios aspectos a tener en cuenta de cara al establecimiento de una posible correlación general (Figs. 2, 3 y 4).

Uno de los primeros hechos observables en las gráficas es que existen numerosos ejemplos en los que el margen altitudinal en el que se enmarca un mismo nivel de terraza es muy amplio; como casos extremos, podrían citarse los valores mencionados para el río Alcanadre y sus terrazas $T_{5}$ (entre $45-96 \mathrm{~m}$ ) o $\mathrm{T}_{6}$ (entre 66-151). Teniendo en cuenta que los valores topográficos suelen ser -como ya hemos mencionado- los 
determinantes para la identificación de los distintos niveles, surge la duda de si realmente se trata de una única superficie o se han comprimido varios niveles dispersos espacialmente en uno sólo. En este sentido parece claro que, en cuanto a 1 número de niveles de terraza se refiere, es evidente que se ha ido registrando con el paso del tiempo una tendencia por parte de los investigadores a incrementar la complejidad de las secuencias de terrazas fluviales identificadas en las distintas cuencas, desde aquellos primeros trabajos en los que se tendía a relacionar el número de acumulaciones con las glaciaciones alpinas, hasta las últimas revisiones en las que, ya desechada la validez de ese referente cronologico en nuestro contexto espacial, se llegan a reconocer más de diez niveles en determinados ríos. Este hecho es un primer factor que condiciona la elaboración de correlaciones generales, ya que muchas veces los datos de partida -en función de las fuentes empleadas- son tan heterogéneos que restan fiabilidad a las conclusiones.

Otro hecho que se evidencia en la representación gráfica de las terrazas de los principales sistemas fluviales de la Depresión del Ebro es que hasta aproximadamente los $60 \mathrm{~m}$ de altitud relativa (rango asignado a las acumulaciones comúnmente clasificadas como bajas y medias), el número de niveles es notoriamente superior al registrado a partir de esta cota (terrazas altas) (Fig. 2, 3 y 4). Así por ejemplo, el sistema del río Ebro, en su sector navarro, integra un total de seis niveles hasta los $60 \mathrm{~m}$, mientras que por encima de esta altura y hasta los $185 \mathrm{~m}$ de su terraza más alta (es decir, en $125 \mathrm{~m}$ de desnivel) únicamente comprende cuatro niveles. Similares circunstancias se registran en las secuencias de los cursos ibéricos, donde en el caso del Najerilla se constatan cinco niveles hasta los $60 \mathrm{~m}$ y otros cinco niveles hasta los $245 \mathrm{~m}$ (en un desnivel de $185 \mathrm{~m}$ ), así como en las de los pirenaicos, destacando el Cinca con cinco niveles hasta los $60 \mathrm{~m}$ y seis niveles hasta los $200 \mathrm{~m}$ (desnivel de 140 $\mathrm{m})$.

Este evidente incremento de diferencia altitudinal entre niveles en relación con la antigüiedad de las acumulaciones parece responder a varias circunstancias muy claras. En primer lugar, el reducido número de niveles antiguos identificados, y la gran diferencia altitudinal existente entre ellos, estarían relacionados: 1) con la existencia de unos procesos de erosión prolongados en el tiempo y selectivos espacialmente (en función sobre todo de factores litológicos locales, con lo que eso conlleva de cara a 1 a conservación o no de niveles a lo largo de la Depresión), procesos que habrían hecho desaparecer los testimonios de terrazas altas en un gran número de interfluvios; 2) con la probable disminución en la capacidad de encajamiento de la red fluvial a lo largo del Cuaternario, ligada a una mayor potencia erosiva inicial de los distintos sistemas fluviales generada por los bruscos reajustes en el nivel de base de la cuenca. En segundo lugar, la mayor abundancia de niveles de edad más reciente, constitutivos de las terrazas medias y bajas, se explicaría recurriendo a argumentos semejantes: 1) por una parte, como es evidente, relacionados con la actuación más reducida en el tiempo de los 
procesos erosivos; 2) por otra, con la respuesta que ofrecen los sistemas fluviales de la Depresión ante su progresivo encajamiento: al aumentar el confinamiento dentro de las llanuras aluviales, los cauces se vuelven mucho más sensibles ante cualquier variación de los factores que integran el sistema (climáticos, estructurales, litológicos, antrópicos, etc.), sensibilidad que se traduce en la mayor alternancia de etapas de agradación e incisión y en la consiguiente génesis de un mayor número de niveles de terraza.

Aparte de la existencia diferenciada de más o menos niveles de terraza según su edad, llama indudablemente la atención el hecho de que la correlación entre niveles acumulativos de los principales ríos de la Depresión del Ebro parece ser significativa únicamente para los niveles más bajos $\left(\mathrm{T}_{1} \mathrm{y} \mathrm{T}_{2}\right)$, es menor en las terrazas medias, y se hace muy escasa conforme aumenta la antigüedad de los depósitos (Figs. 2, 3 y 4). Las hipótesis que acabamos de plantear -sobre todo la existencia de procesos de erosión selectiva- sirven para explicar estas circunstancias, aunque adicionalmente haya otros elementos que potencian la existencia de marcadas diferencias entre cuencas: 1) la propia dinámica del cauce y sus variaciones a lo largo del tiempo: el río Oja, por ejemplo, es uno de los casos más ilustrativos; a partir de la terraza $\mathrm{T}_{4}$ presenta unos niveles con alturas muy por debajo de las de otros ríos vecinos; el funcionamiento de este curso como un sistema braided de amplios abanicos aluviales, así como la probable existencia de varios cursos hasta los episodios correspondientes a la elaboración de la $\mathrm{T}_{5} \circ \mathrm{T}_{6}$, conllevaron un menor encajamiento respecto a otros sistemas próximos más estables; 2) factores externos al sistema fluvial como la tectónica o el diapirismo: in caso donde la influencia de estos factores parece haber condicionado la configuración de las terrazas es el representado por el desdoblamiento de algunas terrazas del río Ebro en el sector navarro; este desdoblamiento se refleja en la existencia entre los $20 \mathrm{y}$ los $55 \mathrm{~m}$ de altitud relativa con respecto al cauce actual de tres niveles de agradación, uno más de lo que es habitual en otros sectores de este mismo curso; 3) por último, un control determinante en algunos sectores ha sido el litológico: en la Depresión del Ebro, el caso más evidente -entre otros- es el del tramo inferior del río Gallego, en el que la disolución del sustrato margo-yesífero ha supuesto el engrosamiento y superposición de varios niveles de terraza, de manera que, en algunos sectores, determinados episodios acumulativos aparecen completamente fosilizados.

A la vista de lo mencionado, es evidente que se hace muy difícil llegar a establecer una correlación entre las secuencias conservadas en cauces distintos a lo largo de toda la Depresión. Al gran número de factores que intervienen en 1 a configuración de las terrazas fluviales -que pueden además actuar de forma diferente en distintos tramos de un mismo valle-, se suma la acción nada despreciable de la erosión, que como hemos visto puede haber hecho desaparecer todos los vestigios de un nivel dentro de una cuenca, o haber conservado secuencias más o menos completas en distintos tramos de un mismo valle. La evidente falta de correlación altitudinal de 
buena parte de los niveles identificados -salvo en el caso ya mencionado de las acumulaciones más bajas- genera, por ejemplo en los cauces del Jalón y el Huerva (aunque esta circunstancia se constata en otros muchos valles), que el nivel $T_{7}$ en la cuenca del Jalón se localice a una altura de $95-110 \mathrm{~m}$, mientras que ese mismo nivel $\mathrm{T}_{7}$ en la cuenca vecina del Huerva aparezca a una altura de $82-87 \mathrm{~m}$; sin disponer de una cronología fiable no se puede asegurar si esos niveles $\mathrm{T}_{7}$ se generaron en una misma fase acumulativa, o bien son restos de etapas distintas: en el primer caso factores locales podrían haber condicionado un comportamiento diferente de los dos sistemas fluviales, produciendo un mayor o menor encajamiento en la secuencia; en el segundo, estaríamos ante un caso de erosión selectiva de los ya mencionados. Este segundo supuesto -que creemos tiene un peso mayor que el que se le concedia hasta ahora, sobre todo para individualizar niveles altos, aislados- supondría incrementar notablemente el número de niveles de terraza localizados en la Depresión del Ebro, hecho que, por otra parte, se correspondería mucho mejor, tanto con las secuencias isotópicas globales, de valor climático, definidas para el Cuaternario desde hace ya varias décadas, como con lo observado en otros ríos peninsulares donde, además del factor climático, intervienen otros elementos que pueden modificar localmente las secuencias.

Aparte de la conservación o no de la totalidad de los niveles de terraza de una secuencia fluvial, y aunque haya podido existir una buena sincronía en la elaboración de las acumulaciones en determinadas cuencas de la Depresión (en aquellos casos en los que los factores implicados en su génesis fueran más o menos coincidentes: cuencas de tamaño similar, con rasgos topográficos, litológicos o tectónicos semejantes, sujetas a procesos climáticos parecidos), no cabe duda que otros cursos fluviales, dentro de un espacio tan amplio como el analizado, han podido evolucionar -bien a lo largo de todo el Cuaternario, bien en fases puntuales del mismo- de forma muy diferente. Este hecho se habría plasmado en la existencia de secuencias complejas, difícilmente correlacionables entre sí, que obligarían a tomar con precaución la posibilidad de establecer una correlación general válida para el ámbito de la Depresión del Ebro.

\section{BIBLIOGRAFÍA}

ALBERTO, F., GUTIÉRREZ, M., IBÁÑEZ, M. J., MACHÍN, J., PEÑA, J. L., POCOVI, J. y RODRIGUEZ, J. (1984): El Cuaternario de la Depresión del Ebro en la región aragonesa. Cartografía y sintesis de los conocimientos existentes. Univ. de Zaragoza-Est. Exp. Aula Dei. 217 p. Zaragoza.

BATALLER, J. R. (1937): "Nota sobre uns dipòsits detritics del Plá d'Urgell", Arxius Esc. Sup. d'Agricultura, t. II (3), 621-633.

BIROT, P. (1937): Recherches sur la morphologie des Pyrénées orientales franco-espagnoles. Tesis Doctoral Univ. de París. Bailliere et Fils, 315 p. París. 
BENITO, G. (1989): Geomorfología de la Cuenca Baja del río Gállego. Tesis Doctoral, Univ. de Zaragoza, 764 p. (Inédita).

BENITO, G., PÉREZ-GONZÁLEZ, A., GUTIÉRREZ, F. y MACHADO, M. J. (1998): "River response to Quaternary subsidence due to evaporite solution (Gállego River, Ebro Basin, Spain)", Geomorphology 22, 243-263.

BOMER, B. (1978): La Bassin de l'Ebre et ses bordures montagneuses. Etude Géomorphologique. Tesis Doctoral Univ. de Caen, 602 p. (Inédita).

BULL, W. B. (1990): "Stream-terrace genesis: implications for soil development." En: KNUEPFER, P. L. K. y McFADDEN, L. D. (Eds.): Soils and Landscape Evolution, 351-367. Elsevier, Londres.

CARBONELL, E., MORA, R. y FULLOLA, J. M. (1987): "Radiografia dels tecno-complexos del Plistocène Superior de la vall de La Femosa (Segriè)". Cypsela, 6, 201-210.

ECHEVERRÍA, M. T. (1984): "El valle del río Aguasvivas. Estudio geomorfológico". Geographicalia, 19-20, 11-95.

FULLOLA, J. M. (1992): "El Paleolítico en Cataluña". En: UTRILLA, P. (Coord.): Aragón/Litoral Mediterráneo. Intercambios culturales durante la Prehistoria. 37-53. Institución Fernando E1 Católico. Zaragoza.

GONZALO, A. (1981): El relieve de La Rioja. Análisis de geomorfología estructural. Instituto de Estudios Riojanos. 508 p. Logroño.

GRACIA, J. (1985): Geomorfología de las Bardenas Orientales. Mem. de Licenciatura Univ. de Zaragoza. 172 p. (Inédita).

IBÁÑEZ, M. J. (1976): El piedemonte ibérico bajoaragonés. Estudio Geomorfológico. Instituto de Geografía Aplicada, C.S.I.C. 523 p. Madrid.

IBARRA, P. (1985): "Geomorfología del Arba de Biel". Medio Geográfico, 2, 59-65.

JULIÁN, A. (1996): Los meandros encajados del río Ebro entre La Zaida y Caspe: Estudio geomorfológico. Grupo Cultural Caspolino-Institución Fernando El Católico, 143 p. Zaragoza.

JULIÁN, A. (1997): Cartografía y correlación general de las acumulaciones cuaternarias de la Depresión del Ebro. Tesis Doctoral, Prensas Universitarias de Zaragoza, Serie microfichas, 579 p. Zaragoza.

LERÁNOZ, B. (1992): "Evolución del curso del río Ebro y de sus afluentes en la Ribera de Navarra (NE de España)". En: LÓPEZ, F., CONESA, C. y ROMERO, M. A. (Eds.): Estudios de Geomorfología en España, Actas II Reunión Nacional de Geomorfología, I, 325-332. S.E.G. Murcia.

LERÁNOZ, B. (1993): Geomorfología y Geología Ambiental de la Ribera de Navarra. Tesis Doctoral Univ. de Zaragoza. 521 p. (Inédita).

LÓPEZ-AGOS, E. (1948): "Restos de Elephas antiquus en el Cerro de Cantabria (Logroño)." Berceo, IX, 592-594. 
LÓPEZ-AGOS, E. (1965): "Nuevos restos de Elephas antiquus Falc. en el Cerro de Cantabria". Berceo, LXXIV, 117.

MACKLIN, M. G. y PASSMORE, D. G. (1995): "Pleistocene environmental change in the Guadalope basin, northeast Spain". En: LEWIN, J., MACKLIN, M. G. y WOODWARD, J. C. (Eds.): Mediterranean Quaternary River Environments. 103-113. Balkema. Rotterdam.

MARTÍ BONO, C. (1977): "Altos valles de los ríos Aragón y Gállego". Trabajos Neógeno Cuaternario, 6. $337-348$.

MAZO, C. y UTRILLA, P. (1992): "Nota sobre dos bifaces amigdaloides de la provincia de Zaragoza". Bol. del Museo de Zaragoza, 11, 5-12.

MENSUA, S. e IBÁÑEZ, M. J. (1977): Sector Central de la Depresión del Ebro. Mapa de terrazas fluviales y glacis. III Reunión Nac. Grupo Esp. Trabajo del Cuaternario. 18 p. Zaragoza.

MORA, R., TERRADAS, J., MARTÍNEZ, J., PARDOS, E. y ROVIRA, M. L. (1992): "Primera aproximación al estudio de las ocupaciones humanas de la Cueva de l'Estret de Tragó (Os de Balaguer, Lérida)". En: UTRILLA, P. (Coord.): Aragón/Litoral Mediterráneo. Intercambios culturales durante la Prehistoria. 97-106. Institución Fernando El Católico. Zaragoza.

OBERMAIER, H. (1925): El hombre fósil. F.C.E.,Madrid.

PATTON, P. C. y SCHUMM, S. A. (1981): "Ephemeral-stream processes: implications for studies of Quaternary valley fills," Quaternary Research, 15, 24-43.

PELLICER, F. (1984): Geomorfología de las Cadenas Ibéricas entre el Jalón y el Moncayo. Cuadernos de Estudios Borjanos, XI-XII, 289 p. Borja (Zaragoza).

PEÑA, J. L. (1983): La Conca de Tremp y Sierras Prepirenaicas comprendidas entre los ríos Segre y Noguera Ribagorzana: Estudio geomorfológico. Instituto de Estudios Ilerdenses. 373 p. Lleida.

PEÑA, J. L. (1988): Las acumulaciones cuaternarias de los Llanos Leridanos. Instituto de Estudios Ilerdenses, 81 p. Lleida.

PEÑA, J. L y SANCHO, C. (1988): "Correlación y evolución cuaternaria del sistema fluvial SegreCinca en su curso bajo (provs. de Lérida y Huesca)". Cuaternario y Geomorfología, 2, 77-83.

PÉREZ-GONZÁLEZ, A. (1989): "Depresión del Duero". En: BIELZA, V. (Coord.): Territorio y Sociedad en España I. Geografía Física, 176-188. Taurus. Madrid.

PÉREZ-GONZÁLEZ, A., CABRA, P. y ANCOCHEA, E. (1988): "Depresión del Tajo". Mapa del Cuaternario en España, 175-186. I.T.G.M.E. Madrid.

PETTS, G. y FOSTER, I. (1985): Rivers and Landscape. Annold. Londres.

RODRÍGUEZ, J. (1986): Geomorfología de las Sierras Exteriores oscenses y su piedemonte. Instituto de Estudios Altoaragoneses, 172 p. Huesca.

SÁEZ, A. (1955): Arqueolítico y Paleolítico. Noticiario Arqueológico Hispánico, III-IV, $217-247$. 
SANCHO, C. (1988): Geomorfología de la Cuenca Baja del río Cinca. Tesis Doctoral Univ. de Zaragoza. 743 p. (Inédita).

SCHUMM, S. A. (1981): "Evolution and response of the fluvial system. Sedimentological implications", Soc. of Economic Paleontologist and Mineralogist, 31, 19-29.

SOLÉ, L. (1957): "Le Quaternaire des Pyrénées." V Congre. Int. INQUA. Livret-guide de l'excursion N1 Pyrénées, 1-28. Madrid.

SORIANO, A. (1986): Geomorfología del piedemonte ibérico en el sector central de la Depresión del Ebro. Tesis Doctoral Univ. de Zaragoza. 359 p.

SORIANO, A. (1990): Geomorfología del sector centromeridional de la Depresión del Ebro. Institución Fernando El Católico, 269 p. Zaragoza.

UTRILLA, P. (1995): "El valle del Ebro durante el Tardiglaciar y comienzos del Holoceno. Las relaciones con el Magdaleniense Cantábrico". En: MOURE, A. y GONZÁLEZ, C. (Eds.): El final del Paleolitico Cantábrico. 281-311. Univ. de Cantabria. Santander.

UTRILLA, P. y MAZO, C. (1991): "Excavación de urgencia en el abrigo de Las Forcas (Graus). Las ocupaciones magdalenienses y epipaleolíticas". Bolskan, 8, 31-78.

UTRILLA, P. y TILO, M.A. (1995): "Cabezo Marañán (Castelserás): un lugar de explotación del silex durante el Paleolítico Medio". Al-Qannis, 4.

YETANO, M. (1978): "La evolución geomorfológica del valle de la Huerva". Geographicalia, 2, 319.

ZUIDAM, R.A. van (1976): Geomorphological development of the Zaragoza region (Spain). I.T.C. 221 p. Enschede.

ZUIDAM, R.A. van, ZUIDAM-CANCELADO, F. I. y DONKER, N. H. W. (1975): "Geomorfología de la gravera Zamoray (Garrapinillos-Zaragoza)", Acta Geológica Hispánica, t. X, 4, 150-159. 